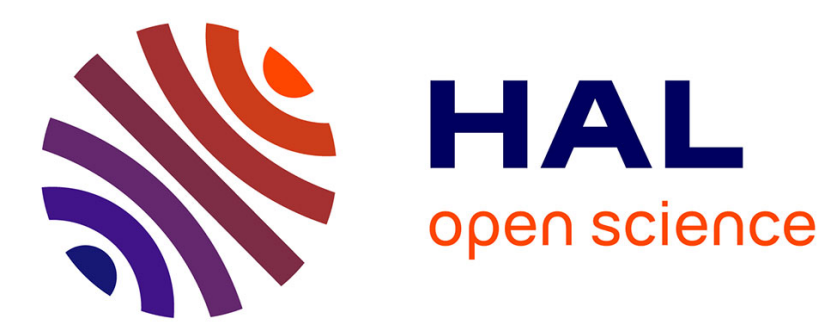

\title{
Guaranteed Voronoi-based Deployment for Multi-Agent Systems under Uncertain Measurements
}

Thomas Chevet, Cristina Stoica Maniu, Cristina Vlad, Youmin Zhang

\section{To cite this version:}

Thomas Chevet, Cristina Stoica Maniu, Cristina Vlad, Youmin Zhang. Guaranteed Voronoi-based Deployment for Multi-Agent Systems under Uncertain Measurements. ECC 2019 - European Control Conference, Jun 2019, Naples, Italy. pp.4016-4021, 10.23919/ECC.2019.8796152 . hal-02100528

\section{HAL Id: hal-02100528 \\ https://hal.science/hal-02100528}

Submitted on 19 Aug 2019

HAL is a multi-disciplinary open access archive for the deposit and dissemination of scientific research documents, whether they are published or not. The documents may come from teaching and research institutions in France or abroad, or from public or private research centers.
L'archive ouverte pluridisciplinaire HAL, est destinée au dépôt et à la diffusion de documents scientifiques de niveau recherche, publiés ou non, émanant des établissements d'enseignement et de recherche français ou étrangers, des laboratoires publics ou privés. 


\title{
Guaranteed Voronoi-based Deployment for Multi-Agent Systems under Uncertain Measurements
}

\author{
Thomas Chevet $^{1}$, Cristina Stoica Maniu ${ }^{1}$, Cristina Vlad ${ }^{1}$ and Youmin Zhang ${ }^{2}$
}

\begin{abstract}
In this paper, a decentralized robust tube-based model predictive control algorithm is used for two-dimensional Voronoi-based deployment of a multi-agent system in a bounded convex area, where the planar motion of each agent is subject to uncertain measurements. A bias bounded by a rectangle is thus considered for each agent's position measurement. The convex area of deployment is then partitioned into guaranteed Voronoi cells separated by a bounded unauthorized corridor. By using a decentralized robust predictive control, each agent is guaranteed to evolve inside the safety region defined by the agent's guaranteed Voronoi cell and to converge to a point in a set centered on the Chebyshev center of this cell, driving the multi-agent system into a static configuration. Simulation results show the effectiveness of the proposed decentralized control strategy on a fleet of quadrotors when one of the agents is subject to a measurement bias due to a sensor fault.
\end{abstract}

\section{INTRODUCTION}

In numerous applications, the problem of deployment of a multi-agent system (MAS) composed of unmanned vehicles over a monitored area is of great interest. These applications range from environmental monitoring to search or rescue operations [1], [2], [3]. This problem has been widely studied and various works rely on the deployment of the MAS inside a dynamic Voronoi partition [4] of the area of interest. Several existing results focus on driving the MAS into a centroidal Voronoi configuration where the target point is the center of mass of the Voronoi cell [5], [6], [7] but this point can be difficult to compute. A simpler objective is to consider the Chebyshev center of the cell [8], [9], [10], i.e. the center of the largest euclidean ball inscribed in the cell. However, these techniques guarantee the MAS convergence into a static configuration under the assumption that the agents' positions are exactly known. When the agents are subject to disturbances, a guaranteed Voronoi diagram [11], [12], taking into account the uncertainty on the position, is used. In this case, a prohibited corridor separates the guaranteed Voronoi cells. The objective is then to reach a static configuration where each agent lies on a target point belonging to a guaranteed cell.

Several control algorithms have been studied for the deployment of a MAS such as consensus based algorithms [13], [1], potential field [2], gradient ascent [12] or model

\footnotetext{
${ }^{1}$ T. Chevet, C. Stoica Maniu and C. Vlad are with Laboratoire des Signaux et Systèmes (L2S), CentraleSupélec-CNRS-Université ParisSud, Université Paris-Saclay, 3, rue Joliot Curie, 91192 Gif-sur-Yvette, France. E-mail: \{thomas.chevet, cristina.stoica, cristina.vlad\}@l2s.centralesupelec.fr

${ }^{2}$ Y. Zhang is with Department of Mechanical, Industrial and Aerospace Engineering, Concordia University, 1455 Blvd. de Maisonneuve, Montréal, QC H3G 1M8, Canada. E-mail: ymzhangdencs. concordia.ca
}

predictive control (MPC) [14], [9], [15]. Moreover, in the case of a Chebyshev center based control algorithm, a statefeedback control law ensures that a static optimized coverage configuration is attained [8], [10]. In order to take into account possible disturbances on the system, robust MPC techniques have been proposed in [16], [17] for a single system, based on tubes of robust positively invariant sets [18], [19] centered on the system's nominal trajectory. With such a control algorithm, the system's state is guaranteed to remain inside the tube. In the context of multi-agent systems, this property is used during the deployment to define safety regions for the considered agents as in [20].

In this paper, a MAS composed of unmanned vehicles for which the position is controlled via the velocity is considered. These agents are deployed inside a two-dimensional convex bounded polyhedron called working space. Each agent is considered to be subject to possible measurement uncertainties on its position, bounded by a rectangle. Using rectangles to bound the uncertainties, quadratic constraints in the MPC problem are avoided. The working space is then partitioned into guaranteed Voronoi cells based on these uncertain rectangles centered on the nominal position of the unmanned vehicles and separated by a prohibited corridor. Then, by using a decentralized robust tube-based MPC law, each agent tracks the Chebyshev center of its guaranteed Voronoi cell. With this control law, the agent's position is constrained to remain inside the guaranteed Voronoi cell. Thus, due to the properties of the tube-based MPC law, each agent remains inside this cell which is then a safety region for collision avoidance during deployment. The contribution of this paper covers: 1) a decentralized tube MPC strategy for the guaranteed Voronoi-based deployment of multi-agent systems with uncertain measurements leading to a bounded unauthorized corridor between the guaranteed Voronoi cells, thus providing anti-collision guarantees; 2) the application of the proposed control strategy on a fleet of quadrotors with measurement bias due to sensor faults.

Section II describes the construction of the guaranteed Voronoi diagram of uncertain rectangles. Section III presents the proposed robust tube-based MPC algorithm for the deployment of the MAS, based on the guaranteed Voronoi diagram of uncertain rectangles. An application of the guaranteed Voronoi deployment framework to a fleet of unmanned aerial vehicles (UAVs) is detailed in Section IV. Finally, concluding remarks and perspectives are drawn in Section V.

Notation. In the following, $\mathbb{R}$ is the set of the real numbers. The matrices $\mathbf{0}_{n \times m}$ and $\mathbf{1}_{n \times m}$ are the matrices of size $n \times m$, with $n$ and $m$ positive integers, filled respectively 
with zeros and ones. The matrix $\boldsymbol{I}_{n}$ is the identity matrix of size $n$. The transpose of the matrix $\boldsymbol{A}$ is denoted by $\boldsymbol{A}^{\top}$. A matrix $\operatorname{diag}\left(a_{1}, \ldots, a_{n}\right)$ is a diagonal matrix with diagonal elements $a_{1}, \ldots, a_{n}$. The spectrum of the matrix $\boldsymbol{A}$ is denoted by $\rho(\boldsymbol{A})$. The vector $|\mathbf{x}|$ contains the absolute value of each element of the vector $\mathbf{x}$. The Euclidean norm of the vector $\mathbf{x}$ is $\|\mathbf{x}\|_{2}^{2}=\mathbf{x}^{\top} \mathbf{x}$. The quadratic norm of the vector $\mathbf{x}$ weighted by the matrix $Q$ is $\|\mathrm{x}\|_{Q}^{2}=\mathrm{x}^{\top} \boldsymbol{Q} \mathrm{x}$, where $Q=Q^{\top}$ is a strictly positive definite matrix, denoted by $Q \succ 0$. The set of integers from $n$ to $m$ is denoted by $\overline{n, m}$. The cardinality of a set $\mathscr{S}$ is denoted by $|\mathscr{S}|$. The Minkowski sum of two sets $\mathscr{A}$ and $\mathscr{B}$ is the set $\mathscr{A} \oplus \mathscr{B}=\{a+b \mid a \in \mathscr{A}, b \in \mathscr{B}\}$ and the Pontryagin difference of these sets is $\mathscr{A} \ominus \mathscr{B}=$ $\{a \in \mathscr{A} \mid a+b \in \mathscr{A}, \forall b \in \mathscr{B}\}$.

\section{Guaranteed Voronoi Diagram of Uncertain RECTANGLES}

\section{A. Preliminaries}

A multi-agent system $\Sigma$ is deployed into a compact convex region $\mathbb{W} \subset \mathbb{R}^{2}$. An agent $\alpha \in \Sigma$ obeys the discretized linear dynamics:

$$
\begin{aligned}
\mathbf{x}_{\alpha}(k+1) & =\mathbf{x}_{\alpha}(k)+T_{s} \mathbf{v}_{\alpha}(k) \\
\mathbf{y}_{\alpha}(k) & =\mathbf{x}_{\alpha}(k)+\mathbf{r}_{\alpha}(k),
\end{aligned}
$$

with $\mathbf{x}_{\alpha}=\left[\begin{array}{ll}x_{\alpha} & y_{\alpha}\end{array}\right]^{\top} \in \mathbb{W}, \mathbf{v}_{\alpha}=\left[\begin{array}{ll}v_{\alpha}^{x} & v_{\alpha}^{y}\end{array}\right]^{\top} \in \mathbb{R}^{2}$ and $\mathbf{y}_{\alpha} \in \mathbb{R}^{2}$ the agent's position, horizontal velocity and output, respectively. Moreover $\mathbf{r}_{\alpha} \in \mathbb{R}^{2}$ is a bounded measurement disturbance and $T_{s}$ is the sampling period of the system. The nominal system associated with (1) for the agent $\alpha \in \Sigma$ is:

$$
\begin{aligned}
\overline{\mathbf{x}}_{\alpha}(k+1) & =\overline{\mathbf{x}}_{\alpha}(k)+T_{s} \overline{\mathbf{v}}_{\alpha}(k) \\
\overline{\mathbf{y}}_{\alpha}(k) & =\overline{\mathbf{x}}_{\alpha}(k) .
\end{aligned}
$$

In order to simplify the reading of this section, the time dependence is further dropped. In the literature, the uncertainty $\mathbf{r}_{\alpha}$ is considered to be bounded either by a circle [12], [11], an ellipse [21] or a polyhedron [11]. However, if $\mathbf{r}_{\alpha}$ is considered to be bounded by a rectangle:

$$
\mathscr{R}_{\alpha}^{0}=\left\{\mathbf{r} \in \mathbb{R}^{2}|| \mathbf{r} \mid \leq\left[\begin{array}{l}
r_{\alpha}^{x} \\
r_{\alpha}^{y}
\end{array}\right]\right\},
$$

with $r_{\alpha}^{x}, r_{\alpha}^{y} \geq 0$, the measurement disturbance is fully encompassed. Thus, the output of the agent $\alpha \in \Sigma$ is guaranteed to belong, at time instant $k$, to $\mathscr{R}_{\alpha}=\left\{\mathbf{x}_{\alpha}\right\} \oplus \mathscr{R}_{\alpha}^{0}$.

\section{B. Guaranteed Voronoi Diagram}

When the positions $\mathbf{x}_{\alpha} \in \mathbb{W}$ of the agents, with $\alpha \in \Sigma$, are well known, the Voronoi diagram [4] of the compact convex set $\mathbb{W}$, in which the multi-agent system is deployed, is:

$$
\mathbb{W}=\bigcup_{\alpha \in \Sigma} \mathbb{V}_{\alpha} \text {, with } \mathbb{V}_{\alpha} \cap \mathbb{V}_{\beta}=\emptyset, \forall \alpha, \beta \in \Sigma, \alpha \neq \beta,
$$

with the Voronoi cells:

$$
\mathbb{V}_{\alpha}=\left\{\mathbf{x} \in \mathbb{W} \mid\left\|\mathbf{x}-\mathbf{x}_{\alpha}\right\|_{2} \leq\left\|\mathbf{x}-\mathbf{x}_{\beta}\right\|_{2}, \forall \beta \neq \alpha\right\} .
$$

However, when the agents' position is uncertain, this definition is modified as presented in [11] or [12]. Given two agents $\alpha, \beta \in \Sigma$ and their associated uncertain regions $\mathscr{R}_{\alpha}$ and $\mathscr{R}_{\beta}$, the set of the points closer to $\mathscr{R}_{\alpha}$ than to $\mathscr{R}_{\beta}$ can be defined as follows:

$\mathscr{H}_{\alpha}^{\beta}=\left\{\mathbf{x} \in \mathbb{W} \mid\|\mathbf{x}-\mathbf{y}\|_{2} \leq\|\mathbf{x}-\mathbf{z}\|_{2}, \forall \mathbf{y} \in \mathscr{R}_{\alpha}, \mathbf{z} \in \mathscr{R}_{\beta}\right\}$.

The guaranteed Voronoi $(\mathrm{GV})$ cell with respect to the considered uncertain measurements of the agent $\alpha \in \Sigma$ is then:

$$
\mathbb{V}_{\alpha}^{g}=\bigcap_{\substack{\beta \in \Sigma \\ \beta \neq \alpha}} \mathscr{H}_{\alpha}^{\beta}
$$

and the GV diagram is now such that:

$$
\bigcup_{\alpha \in \Sigma} \mathbb{V}_{\alpha}^{g} \subseteq \mathbb{W} \text {, with } \mathbb{V}_{\alpha}^{g} \cap \mathbb{V}_{\beta}^{g}=\emptyset, \forall \alpha, \beta \in \Sigma, \alpha \neq \beta \text {. }
$$

In the following, the expressions guaranteed Voronoi cell or guaranteed Voronoi cell of uncertain rectangles will always designate a cell constructed by considering rectangle uncertainties.

\section{Cell Boundary}

For two uncertain regions $\mathscr{R}_{\alpha}$ and $\mathscr{R}_{\beta}$, the boundary of the set $\mathscr{H}_{\alpha}^{\beta}$ is the set:

$$
\mathscr{B}_{\alpha}^{\beta}=\left\{\mathbf{x} \in \mathbb{W} \mid \max _{\mathbf{y} \in \mathscr{R}_{\alpha}}\|\mathbf{x}-\mathbf{y}\|_{2}=\min _{\mathbf{z} \in \mathscr{R}_{\beta}}\|\mathbf{x}-\mathbf{z}\|_{2}\right\} .
$$

Consider $\mathbf{x}=\left[\begin{array}{ll}x & y\end{array}\right]^{\top}$ and let $\mathbf{x}_{\alpha}, \mathbf{x}_{\beta}$ be the centers of the sets $\mathscr{R}_{\alpha}$ and $\mathscr{R}_{\beta}$, respectively. Then, the following expressions hold for all $\mathbf{x} \in \mathbb{W}$ :

$$
\begin{aligned}
& \max _{\mathbf{y} \in \mathscr{R}_{\alpha}}\|\mathbf{x}-\mathbf{y}\|_{2}^{2}=\left(\left|x-x_{\alpha}\right|+r_{\alpha}^{x}\right)^{2}+\left(\left|y-y_{\alpha}\right|+r_{\alpha}^{y}\right)^{2} \\
& \min _{\mathbf{z} \in \mathscr{R}_{\beta}}\|\mathbf{x}-\mathbf{z}\|_{2}^{2}=(\left.\max \left\{\left|x-x_{\beta}\right|-r_{\beta}^{x}, 0\right\}\right)^{2} \\
&+\left(\max \left\{\left|y-y_{\beta}\right|-r_{\beta}^{y}, 0\right\}\right)^{2} .
\end{aligned}
$$

Given these two distances, the equality in (8) defines the edge of $\mathscr{H}_{\alpha}^{\beta}$. This boundary is then continuous and composed of line segments and parabolic arcs, having for focus one of the vertices of $\mathscr{R}_{\alpha}$. Due to this last statement, $\mathscr{R}_{\alpha}$ lies in the convex part of the parabolas inducing the parabolic arcs. Thus, the region $\mathscr{H}_{\alpha}^{\beta}$ is convex [22].

Figure 1 illustrates an example of a compact convex region $\mathbb{W}$ with two agents having for positions $\mathbf{x}_{1}$ and $\mathbf{x}_{2}$ and their associated uncertain rectangles $\mathscr{R}_{1}$ and $\mathscr{R}_{2}$. The boundaries $\mathscr{H}_{1}^{2}$ and $\mathscr{H}_{2}^{1}$ delimit the GV cells (the red filled areas). An empty corridor (the white area) appears due to the position measurement uncertainties. The dashed lines are the parabolic arcs mentioned before. However, the GV cells are meant to be used as safety regions for the agents and as position constraints in a MPC optimization problem defined in Section III-B. Thus, the parabolic arcs are difficult to use because they induce quadratic constraints within this optimization problem. Since the set $\mathscr{R}_{\alpha}$ is in the convex part of the parabolas, an inner approximation of each parabolic arc will be further built by the chords joining the two ends of the succession of parabolic arcs instead of the parabolic arcs themselves. Thus, the border of $\mathscr{H}_{\alpha}^{\beta}$ is only composed of 


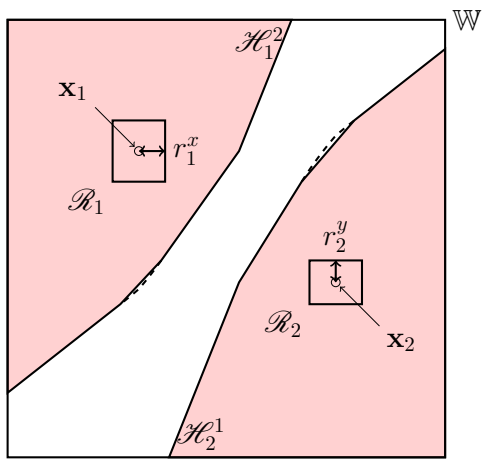

Fig. 1. Example of guaranteed Voronoi diagram for two agents with uncertain positions bounded by rectangles.

line segments and the $\mathrm{GV}$ cell $\mathbb{V}_{\alpha}^{g}$ is then a bounded convex polytope. Notice that this trade-off between the complexity and the accuracy of the results does not affect the guaranteed Voronoi partition.

\section{Proposed Deployment Control}

A. Robust Tube-Based MPC for an Agent subject to Uncertain Position Measurements

The proposed control strategy for the multi-agent system deployment is based on a robust tube-based MPC [16]. Each agent $\alpha \in \Sigma$ follows the dynamics from (1) with an uncertainty on the position measurement. Such a control law is used to guarantee that the uncertain state of the system (1) stays in a tube centered on the nominal state of (2). The control input is then:

$$
\mathbf{v}_{\alpha}(k)=\overline{\mathbf{v}}_{\alpha}(k)+\boldsymbol{K}_{\alpha}\left(\widehat{\mathbf{x}}_{\alpha}(k)-\overline{\mathbf{x}}_{\alpha}(k)\right),
$$

where $\overline{\mathbf{v}}_{\alpha}(k)$ is obtained via a MPC algorithm applied on the nominal system (2) that will be described in Section III$\mathrm{B}, \widehat{\mathbf{x}}_{\alpha}(k)$ and $\overline{\mathbf{x}}_{\alpha}(k)$ are respectively the estimated and the nominal positions of the agent $\alpha$ and $\boldsymbol{K}_{\alpha} \in \mathbb{R}^{2 \times 2}$ is a statefeedback gain. To estimate the state of the uncertain system (1), a discrete-time Luenberger observer is used having for gain matrix $\boldsymbol{L}_{\alpha} \in \mathbb{R}^{2 \times 2}$ :

$$
\begin{aligned}
\widehat{\mathbf{x}}_{\alpha}(k+1) & =\widehat{\mathbf{x}}_{\alpha}(k)+T_{s} \mathbf{v}_{\alpha}(k)+\boldsymbol{L}_{\alpha}\left(\mathbf{y}_{\alpha}(k)-\widehat{\mathbf{y}}_{\alpha}(k)\right) \\
\widehat{\mathbf{y}}_{\alpha}(k) & =\widehat{\mathbf{x}}_{\alpha}(k) .
\end{aligned}
$$

The estimation error between the real and the estimated position $\widetilde{\mathbf{x}}_{\alpha}(k)=\mathbf{x}_{\alpha}(k)-\widehat{\mathbf{x}}_{\alpha}(k)$ is such that:

$$
\widetilde{\mathbf{x}}_{\alpha}(k+1)=\left(\boldsymbol{I}_{2}-\boldsymbol{L}_{\alpha}\right) \widetilde{\mathbf{x}}_{\alpha}(k)-\boldsymbol{L}_{\alpha} \mathbf{r}_{\alpha}(k),
$$

with the spectrum $\rho\left(\boldsymbol{I}_{2}-\boldsymbol{L}_{\alpha}\right)<1$. The deviation between the estimated and the nominal state $\mathbf{e}_{\alpha}(k)=\widehat{\mathbf{x}}_{\alpha}(k)-\overline{\mathbf{x}}_{\alpha}(k)$ is such that:

$\mathbf{e}_{\alpha}(k+1)=\left(\boldsymbol{I}_{2}+T_{s} \boldsymbol{K}_{\alpha}\right) \mathbf{e}(k)+\boldsymbol{L}_{\alpha}\left(\widetilde{\mathbf{x}}_{\alpha}(k)+\mathbf{r}_{\alpha}(k)\right)$,

with $\rho\left(\boldsymbol{I}_{2}+T_{s} \boldsymbol{K}_{\alpha}\right)<1$. Both the gains $\boldsymbol{K}_{\alpha}$ and $\boldsymbol{L}_{\alpha}$ can be obtained by using a pole placement technique or by linear quadratic design.

Based on the definitions of $\widetilde{\mathbf{x}}_{\alpha}$ and $\mathbf{e}_{\alpha}$, the position of the system (1) is such that:

$$
\mathbf{x}_{\alpha}(k)=\overline{\mathbf{x}}_{\alpha}(k)+\widetilde{\mathbf{x}}_{\alpha}(k)+\mathbf{e}_{\alpha}(k)
$$

Definition 1 ([18]): A set $\mathscr{S}$ is called robustly positively invariant (RPI) for the discrete-time system:

$$
\begin{gathered}
\mathbf{x}(k+1)=\boldsymbol{A} \mathbf{x}(k)+\mathbf{w}(k) \\
\text { if } \mathbf{x}(k) \in \mathscr{S}, \forall \mathbf{x}(0) \in \mathscr{S}, \mathbf{w}(k) \in \mathscr{W}, k \geq 0 \text {, or if: }
\end{gathered}
$$

$$
\boldsymbol{A} \mathscr{S} \oplus \mathscr{W} \subseteq \mathscr{S}
$$

Definition 2 ([18]): A set is called minimal robustly positively invariant (mRPI) for (1) if it is a RPI set contained in every RPI set for (1).

Since for systems (13) and (14) the spectra satisfy $\rho\left(\boldsymbol{I}_{2}-\boldsymbol{L}_{\alpha}\right)<1$ and $\rho\left(\boldsymbol{I}_{2}+T_{s} \boldsymbol{K}_{\alpha}\right)<1$, there exists two sets $\widetilde{\mathbb{S}}$ and $\overline{\mathbb{S}}$ that are RPI [19] respectively for (13) and (14). It was proven in [16] that if $\widetilde{\mathbf{x}}_{\alpha}(0) \in \widetilde{\mathbb{S}}\left(\right.$ resp. $\left.\mathbf{e}_{\alpha}(0) \in \overline{\mathbb{S}}\right)$, then $\widetilde{\mathbf{x}}_{\alpha}(k) \in \widetilde{\mathbb{S}}$ (resp. $\mathbf{e}_{\alpha}(k) \in \overline{\mathbb{S}}$ ) for all admissible disturbance sequences and $k \geq 0$. Then, by extension, with the control law (11), the position of the agent $\alpha$ satisfies:

$$
\mathbf{x}_{\alpha}(k) \in\left\{\overline{\mathbf{x}}_{\alpha}(k)\right\} \oplus \widetilde{\mathbb{S}} \oplus \overline{\mathbb{S}} .
$$

\section{B. Decentralized MPC Algorithm for the Deployment of a} MAS subject to Uncertain Position Measurements

The decentralized model predictive controller proposed for the nominal system (2) is derived from the technique presented in [9]. The nominal speed $\overline{\mathbf{v}}_{\alpha}$ for system (2) is given by the optimization problem:

$$
\begin{aligned}
& \min _{\overline{\mathbf{v}}_{\alpha}} \quad \sum_{l=0}^{N_{p}-1}\left(\left\|\overline{\mathbf{x}}_{\alpha}(k+l)-\mathbf{x}_{\alpha}^{\mathrm{obj}}(k)\right\|_{\boldsymbol{Q}}^{2}+\left\|\overline{\mathbf{v}}_{\alpha}(k+l)\right\|_{\boldsymbol{R}}^{2}\right) \\
&+\left\|\overline{\mathbf{x}}_{\alpha}\left(k+N_{p}\right)-\mathbf{x}_{\alpha}^{\mathrm{obj}}(k)\right\|_{\boldsymbol{P}}^{2} \\
& \text { s.t. } \quad \overline{\mathbf{x}}_{\alpha}(k+l+1)=\overline{\mathbf{x}}_{\alpha}(k+l)+T_{s} \overline{\mathbf{v}}_{\alpha}(k+l), \\
& \overline{\mathbf{x}}_{\alpha}(k+l) \in \overline{\mathbb{X}} \\
& \overline{\mathbf{v}}_{\alpha}(k+l) \in \overline{\mathbb{U}} \\
& \overline{\mathbf{x}}_{\alpha}\left(k+N_{p}\right) \in \overline{\mathbb{X}}^{\lambda} .
\end{aligned}
$$

In the cost function of the problem (19), the weighting matrices $\boldsymbol{Q}=\boldsymbol{Q}^{\top}, \boldsymbol{R}=\boldsymbol{R}^{\top} \succ 0$ are diagonal matrices and $\boldsymbol{P}$ is the solution of a discrete-time Riccati equation. The integer $N_{p}>0$ is the prediction horizon.

In the optimization problem (19), the objective point $\mathbf{x}_{\alpha}^{\mathrm{obj}}(k)$ is the Chebyshev center of the guaranteed Voronoi cell $\mathbb{V}_{\alpha}^{g}(k)$ of the agent $\alpha \in \Sigma$. At each time the MPC algorithm is run, all the agents $\alpha \in \Sigma$ broadcast their nominal position $\overline{\mathbf{x}}_{\alpha}$ and their uncertain rectangle $\mathscr{R}_{\alpha}^{0}$. Then, even when the actual bias on the position measurement in (1) is null, each agent constructs its guaranteed cell based on the uncertain rectangles $\overline{\mathscr{R}}_{\alpha}=\left\{\overline{\mathbf{x}}_{\alpha}\right\} \oplus \mathscr{R}_{\alpha}^{0}$ to account for the noise and the model error. Since the agents evolve in the set $\mathbb{W}$, the GV cells are time-varying. The Chebyshev center of $\mathbb{V}_{\alpha}^{g}(k)$ is the center of the largest ball $\mathbb{B}_{\alpha}=$ $\left\{\mathbf{x} \in \mathbb{V}_{\alpha}^{g}(k) \mid\left\|\mathbf{x}-\mathbf{x}_{\alpha}^{\mathrm{obj}}\right\|_{2} \leq \rho_{\alpha}\right\}$ inscribed in $\mathbb{V}_{\alpha}^{g}(k)$ and is 
the solution of the optimization problem:

$$
\begin{array}{cl}
\max _{\mathbf{x}_{\alpha}^{\text {obj }}, \rho_{\alpha}} & \rho_{\alpha} \\
\text { s.t. } & \rho_{\alpha} \geq 0, \\
& \theta_{i}^{\alpha} \geq \boldsymbol{h}_{i}^{\alpha \top} \mathbf{x}_{\alpha}^{\text {obj }}+\left\|\boldsymbol{h}_{i}^{\alpha}\right\| \rho_{\alpha}, i \in \overline{1, n},
\end{array}
$$

with $\mathbb{V}_{\alpha}^{g}(k)=\left\{\mathbf{x} \in \mathbb{W} \mid \boldsymbol{h}_{i}^{\alpha \top} \mathbf{x} \leq \theta_{i}^{\alpha}, i \in \overline{1, n}\right\}, \boldsymbol{h}_{i}^{\alpha} \in \mathbb{R}^{2}$, $\theta_{i}^{\alpha} \in \mathbb{R}$ and the integer $n>0$ the number of sides of $\mathbb{V}_{\alpha}^{g}(k)$.

The set of state constraints $\overline{\mathbb{X}}$ is the set $\mathbb{V}_{\alpha}^{g}(k) \ominus(\widetilde{\mathbb{S}} \oplus \overline{\mathbb{S}})$ and the set of input constraints $\overline{\mathbb{U}}$ is $\mathbb{U} \ominus \boldsymbol{K}_{\alpha} \overline{\mathbb{S}}$, where $\mathbb{U}=\left\{\mathbf{v} \in \mathbb{R}^{2}|| \mathbf{v} \mid \leq\left[\begin{array}{ll}v_{x}^{\max } & v_{y}^{\max }\end{array}\right]^{\top}\right\}$ is a rectangle of hard constraints on $\mathbf{v}_{\alpha}$.

The final set $\overline{\mathbb{X}}^{\dot{\lambda}}$ is a contracted version of the set $\overline{\mathbb{X}}$ based on a contracted version of the GV cell:

$$
\overline{\mathbb{X}}^{\lambda}=\underbrace{\left\{\mathbf{x}_{\alpha}^{\text {obj }}\right\} \oplus \lambda\left(\mathbb{V}_{\alpha}^{g} \oplus\left\{-\mathbf{x}_{\alpha}^{\text {obj }}\right\}\right)}_{\text {contracted Voronoi cell }} \ominus(\widetilde{\mathbb{S}} \oplus \overline{\mathbb{S}}),
$$

with $\lambda \in[0,1)$. The constraint (19e) ensures that $\overline{\mathbb{X}}^{\lambda}$ is a controlled $\lambda$-contractive set [18] for system (2). Then, the nominal system converges to a static Chebyshev configuration [8], [10], where $\overline{\mathbf{x}}_{\alpha}(k+1)=\overline{\mathbf{x}}_{\alpha}(k)=\mathbf{x}_{\alpha}^{\text {obj }}$.

Finally, it was shown in [16] that, with the control law given in (11), the real position $\mathbf{x}_{\alpha}$ and speed $\mathbf{v}_{\alpha}$ of the agent $\alpha \in \Sigma$ satisfy the constraints $\mathbf{x}_{\alpha} \in \mathbb{X}$ and $\mathbf{v}_{\alpha} \in \mathbb{U}$ provided that $\widetilde{\mathbf{x}}_{\alpha}(0) \in \widetilde{\mathbb{S}}$ and $\mathbf{e}_{\alpha}(0) \in \overline{\mathbb{S}}$. Then, with the chosen constraints and with the robust tube-based MPC control algorithm, the real position of each agent is guaranteed to stay inside its GV cell which is a safety region.

\section{Application to a Fleet of UAVs}

\section{A. UAV Model}

Let $\Sigma$ be a system composed of $|\Sigma|$ quadrotor UAVs. A non-linear continuous-time state-space model of such a UAV can be derived from Lagrangian mechanics and a description of this model can be found in [23], [9]. The considered state-space is $\mathbf{X}_{\alpha}=$ $\left[\begin{array}{llllllllllll}x_{\alpha} & y_{\alpha} & z_{\alpha} & \phi_{\alpha} & \theta_{\alpha} & \psi_{\alpha} & v_{\alpha}^{x} & v_{\alpha}^{y} & v_{\alpha}^{z} & \omega_{\alpha}^{x} & \omega_{\alpha}^{y} & \omega_{\alpha}^{z}\end{array}\right]^{\top}$, with $\alpha \in \Sigma$. The signification of all the state variables is gathered in Table I. This model is then linearized around an equilibrium point that corresponds to a hovering state $\mathbf{X}_{\alpha}^{\mathrm{eq}}=$ $\left[\begin{array}{llll}x_{\alpha}^{\mathrm{eq}} & y_{\alpha}^{\mathrm{eq}} & z_{\alpha}^{\mathrm{eq}} & \mathbf{0}_{1 \times 9}\end{array}\right]^{\top}$ leading to a linear state-space model:

$$
\dot{\mathbf{X}}_{\alpha}=\boldsymbol{A}\left(\mathbf{X}_{\alpha}-\mathbf{X}_{\alpha}^{\mathrm{eq}}\right)+\boldsymbol{B} \mathbf{u}_{\alpha}
$$

where $\mathbf{u}_{\alpha}=\mathbf{U}_{\alpha}-\mathbf{U}_{\alpha}^{\text {eq }}$, with $\mathbf{U}_{\alpha}=\left[\begin{array}{llll}u_{\alpha}^{1} & u_{\alpha}^{2} & u_{\alpha}^{3} & u_{\alpha}^{4}\end{array}\right]^{\top}$ the duty cycles of the pulse width modulation voltages supplied to the UAV's motors and $\mathbf{U}_{\alpha}^{\mathrm{eq}}=\frac{m g}{4 K} \mathbf{1}_{4 \times 1}$ the input value to maintain the UAV in hovering state.

In the following, all state variables will be considered to evolve around their equilibrium values. The system (22) is separated into five subsystems to apply the control strategy described in the previous section. The position subsystem is:

$$
\left[\begin{array}{c}
\dot{x}_{\alpha} \\
\dot{y}_{\alpha}
\end{array}\right]=\left[\begin{array}{c}
v_{\alpha}^{x} \\
v_{\alpha}^{y}
\end{array}\right]
$$

TABLE I

NOMENCLATURE AND NUMERICAL VALUES

\begin{tabular}{ll}
\hline$x_{\alpha}, y_{\alpha}, z_{\alpha}$ & UAV's center of mass coordinates \\
$\phi_{\alpha}, \theta_{\alpha}, \psi_{\alpha}$ & Roll, pitch and yaw angles \\
$v_{\alpha}^{x}, v_{\alpha}^{y}, v_{\alpha}^{z}$ & Linear speed of the UAV \\
$\omega_{\alpha}^{x}, \omega_{\alpha}^{y}, \omega_{\alpha}^{z}$ & Angular speed of the UAV \\
$m=1.4 \mathrm{~kg}$ & UAV's mass \\
$I_{x}=I_{y}=0.03 \mathrm{~kg} \cdot \mathrm{m}^{2}$ & Moments of inertia along \\
$I_{z}=0.04 \mathrm{~kg} \cdot \mathrm{m}^{2}$ & $x, y$ and $z$ directions \\
$L=0.2 \mathrm{~m}$ & Arm's length \\
$C=4 \mathrm{~m}$ & Thrust to moment ratio \\
$K=12 \mathrm{~N} \cdot \mathrm{V}^{-1}$ & Motor gain \\
$g=9.81 \mathrm{~m} \cdot \mathrm{s}^{-2}$ & Gravitational acceleration \\
\hline
\end{tabular}

which provides the references to the pitch/roll subsystems:

$$
\begin{gathered}
{\left[\begin{array}{c}
\dot{v}_{\alpha}^{x} \\
\dot{\theta}_{\alpha} \\
\dot{\omega}_{\alpha}^{y}
\end{array}\right]=\left[\begin{array}{ccc}
0 & g & 0 \\
0 & 0 & 1 \\
0 & 0 & 0
\end{array}\right]\left[\begin{array}{c}
v_{\alpha}^{x} \\
\theta_{\alpha} \\
\omega_{\alpha}^{y}
\end{array}\right]+\frac{K L}{I_{y}}\left[\begin{array}{cccc}
0 & 0 & 0 & 0 \\
0 & 0 & 0 & 0 \\
0 & 0 & 1 & -1
\end{array}\right] \mathbf{u}_{\alpha}^{\theta},} \\
{\left[\begin{array}{c}
\dot{v}_{\alpha}^{y} \\
\dot{\phi}_{\alpha} \\
\dot{\omega}_{\alpha}^{x}
\end{array}\right]=\left[\begin{array}{ccc}
0 & -g & 0 \\
0 & 0 & 1 \\
0 & 0 & 0
\end{array}\right]\left[\begin{array}{c}
v_{\alpha}^{y} \\
\phi_{\alpha} \\
\omega_{\alpha}^{x}
\end{array}\right]+\frac{K L}{I_{x}}\left[\begin{array}{cccc}
0 & 0 & 0 & 0 \\
0 & 0 & 0 & 0 \\
1 & -1 & 0 & 0
\end{array}\right] \mathbf{u}_{\alpha}^{\phi},}
\end{gathered}
$$

and, finally, the altitude and yaw subsystems are:

$$
\begin{gathered}
{\left[\begin{array}{c}
\dot{z}_{\alpha} \\
\dot{v}_{\alpha}^{z}
\end{array}\right]=\left[\begin{array}{cc}
0 & 1 \\
0 & 0
\end{array}\right]\left[\begin{array}{c}
z_{\alpha} \\
v_{\alpha}^{z}
\end{array}\right]+\frac{K}{m}\left[\begin{array}{cccc}
0 & 0 & 0 & 0 \\
1 & 1 & 1 & 1
\end{array}\right] \mathbf{u}_{\alpha}^{z},} \\
{\left[\begin{array}{c}
\dot{\psi}_{\alpha} \\
\dot{\omega}_{\alpha}^{z}
\end{array}\right]=\left[\begin{array}{cc}
0 & 1 \\
0 & 0
\end{array}\right]\left[\begin{array}{c}
\psi_{\alpha} \\
\omega_{\alpha}^{z}
\end{array}\right]+\frac{K C}{I_{z}}\left[\begin{array}{cccc}
0 & 0 & 0 & 0 \\
-1 & -1 & 1 & 1
\end{array}\right] \mathbf{u}_{\alpha}^{\psi} .}
\end{gathered}
$$

All the numerical values of the parameters are given in Table I. The position subsystem (23) is sampled at $T_{s}=0.2$ s. If the position measurement is subject to a bias, the discrete-time version of the system (23) is identical to (1) and the tube-based MPC strategy under uncertain measurements described in Section III is used to control it.

The systems (24) to (27) are sampled at $T_{s}^{\prime}=1 \mathrm{~ms}$. For these systems, the output is equal to the system's state. For each of them, a discrete-time linear quadratic regulator [24] is designed with the weights:

$$
\left\{\begin{aligned}
\boldsymbol{Q}_{z} & =\boldsymbol{I}_{2}, & \boldsymbol{R}_{z} & =1000, \\
\boldsymbol{Q}_{\psi} & =\operatorname{diag}(1,0), & \boldsymbol{R}_{\psi} & =100, \\
\boldsymbol{Q}_{\theta, \phi} & =\operatorname{diag}(1,1,0), & \boldsymbol{R}_{\theta, \phi} & =10 .
\end{aligned}\right.
$$

The total control signal is then:

$$
\mathbf{u}_{\alpha}(k)=\mathbf{u}_{\alpha}^{z}(k)+\mathbf{u}_{\alpha}^{\psi}+\mathbf{u}_{\alpha}^{\theta}+\mathbf{u}_{\alpha}^{\phi}+u_{\alpha}^{\text {angle }} \mathbf{1}_{4 \times 1} .
$$

The term $u_{\alpha}^{\text {angle }}$ is meant to compensate the loss of upward thrust caused by the UAV's inclination. The total upward thrust at time $k$ is $T(k)=m g \cos \theta(k) \cos \phi(k)$ and the objective is then to have $T(k)+4 K u_{\alpha}^{\text {angle }}(k)=m g$.

\section{B. Simulation Scenario}

A multi-agent system composed of 4 quadrotor UAVs is deployed inside a square of $10 \mathrm{~m}$ side length $\mathbb{W}=$ $\left\{\mathbf{x} \in \mathbb{R}^{2}|| \mathbf{x} \mid \leq 5 \cdot \mathbf{1}_{2 \times 1}\right\}$. The position sensor of each agent is considered to be subject to a bias bounded by a square of 0.4 m side length $\mathscr{R}_{\alpha}^{0}=\left\{\mathbf{x} \in \mathbb{R}^{2}|| \mathbf{x} \mid \leq 0.2 \cdot \mathbf{1}_{2 \times 1}\right\}, \forall \alpha \in$ $\overline{1,4}$. 
For each agent, the state observer for the position subsystem (1) is obtained by pole placement such that $\rho\left(\boldsymbol{I}_{2}-\boldsymbol{L}_{\alpha}\right)=\{0,0\}$ and $\boldsymbol{L}_{\alpha}=\boldsymbol{I}_{2}, \forall \alpha \in \overline{1,4}$.

With the state observer described before, a mRPI set for (13) is immediately $\widetilde{\mathbb{S}}=\mathscr{R}_{\alpha}^{0}$ from Definitions 1 and 2. However, for the construction of $\overline{\mathbb{S}}$, a more complex method must be employed. First, the gain $\boldsymbol{K}_{\alpha}$ in (11) is computed by using the method from [17]. This method, if feasible, ensures that the sets $\mathbb{W} \ominus(\widetilde{\mathbb{S}} \oplus \overline{\mathbb{S}}), \mathbb{U} \ominus \boldsymbol{K}_{\alpha} \overline{\mathbb{S}}$, with $\alpha \in \overline{1,4}$ and $\mathbb{U}=\left\{\mathbf{v} \in \mathbb{R}^{2}|| \mathbf{v} \mid \leq 5 \cdot \mathbf{1}_{2 \times 1}\right\}$, are not empty (by using the notations from [17], the tuning parameters used for this method are $\lambda=0.1$ and $\rho=0.57)$. Then, if the obtained gain $\boldsymbol{K}_{\alpha}$ ensures that (14) is stable, an approximation of the mRPI set $\overline{\mathbb{S}}$ is computed by following the algorithm from [25]. Both the sets $\widetilde{\mathbb{S}}$ and $\overline{\mathbb{S}}$, as well as $\boldsymbol{K}_{\alpha} \overline{\mathbb{S}}$, which are the same for each agent, are presented on Fig. 2.

Then, each agent starts from a feasible random position in $\mathbb{W}$. Here, feasible means that $\overline{\mathbf{x}}_{\alpha}(0) \in \mathbb{V}_{\alpha}^{g}(0) \ominus(\widetilde{\mathbb{S}} \oplus \overline{\mathbb{S}})$. At $t=0 \mathrm{~s}$, their position sensors are not subject to any bias. However, as mentioned in Section III-B an uncertainty bounded by a square of $0.4 \mathrm{~m}$ side length is always considered for the construction of the GV diagram. Thus, the guaranteed Voronoi diagram is computed and each agent starts tracking the Chebyshev center of its guaranteed cell. At each time instant the MPC runs, each agent computes its GV cell and its new Chebyshev center. At $t=13 \mathrm{~s}$, agent 4 is subject to a sensor fault leading to a constant bias $\mathbf{r}_{4}=\left[\begin{array}{ll}-0.2 & 0.2\end{array}\right]^{\top}$ on the output of its position sensor.

During the simulation, for each agent $\alpha \in \overline{1,4}$, the setpoints for $z_{\alpha}, v_{\alpha}^{z}$, the angles and the angular speeds are respectively $5 \mathrm{~m}, 0 \mathrm{~m} . \mathrm{s}^{-1}, 0 \mathrm{rad}$ and $0 \mathrm{rad} . \mathrm{s}^{-1}$. The speed references for subsystems (24) and (25) are provided by the position subsystem (23). To limit the values of the pitch and roll angles and remain in a linear domain for the evolution of the system, a rate limitation of $0.2 \mathrm{~m} . \mathrm{s}^{-1}$ on each axis is added on the variation of the objective point $\mathbf{x}_{\alpha}^{\mathrm{obj}}$.

For all the MPC algorithms, the considered tuning parameters are $\boldsymbol{Q}=10 \boldsymbol{I}_{2}, \boldsymbol{R}=\boldsymbol{I}_{2}$ and $N_{p}=5$. The contraction factor for the constraint (19e) is chosen to be $\lambda=0.95$. The optimization solver for the MPC is generated with CVXGEN [26] and the polyhedrons are computed with MPT3 [27].

\section{Simulation Results}

In this simulation scenario, the dynamical model for each agent is the nonlinear model that can be found in [9].

The evolution of the position $\mathbf{x}_{\alpha}$ of all the 4 agents is presented in Fig. 3 as well as the evolution of the nominal position $\overline{\mathbf{x}}_{\alpha}$ and the Chebyshev center $\mathbf{x}_{\alpha}^{\text {obj }}$ of all these agents. This figure also shows the final (at $t=30 \mathrm{~s}$ ) GV diagram (the blue filled sets) as well as the final sets $\mathbb{V}_{\alpha}^{g} \ominus(\widetilde{\mathbb{S}} \oplus \overline{\mathbb{S}})$, with $\alpha \in \overline{1,4}$, represented by the dashed lines. It can be seen that the position of each agent converges to its nominal value which converges to the Chebyshev center of the guaranteed Voronoi cell, except for the agent 4 due to the bias on the measurement. However, the position of agent 4 converges to a point inside $\left\{\overline{\mathbf{x}}_{4}\right\} \oplus \widetilde{\mathbb{S}} \oplus \overline{\mathbb{S}} \subseteq \mathbb{V}_{4}^{g}$ as detailed in (18).

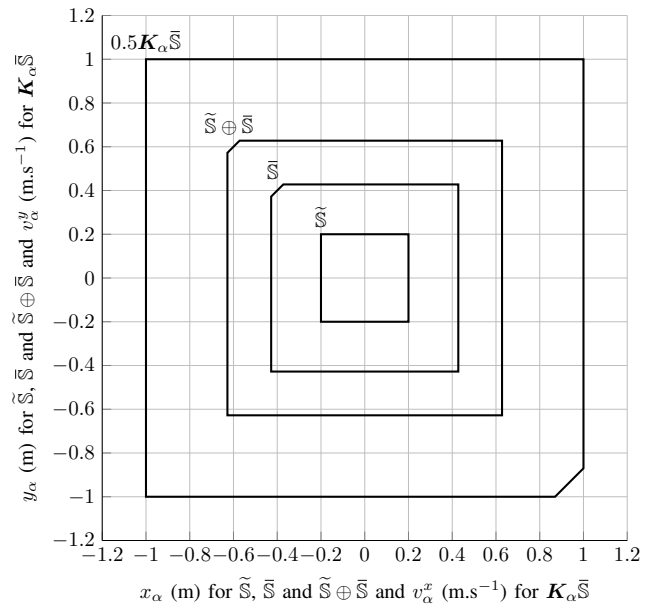

Fig. 2. Approximations of minimum robustly positively invariant sets for the control problem (the set $\boldsymbol{K}_{\alpha} \overline{\mathbb{S}}$ is scaled to fit in the figure).

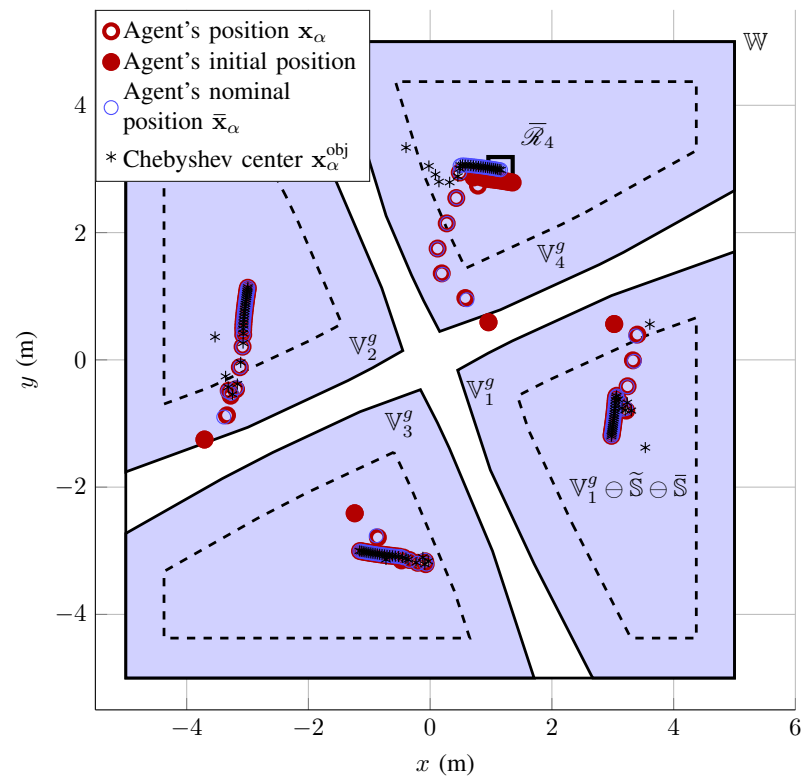

Fig. 3. Evolution of the agents' real positions $\mathbf{x}_{\alpha}$, nominal positions $\overline{\mathbf{x}}_{\alpha}$ and Chebyshev centers $\mathbf{x}_{\alpha}^{\text {obj }}$.

This behavior is illustrated in Fig. 4. The nominal positions $\overline{\mathbf{x}}_{2}$ and $\overline{\mathbf{x}}_{4}$ of the agents 2 and 4 converge to the Chebyshev center of their associated cell. The same can be said for the real position $\mathrm{x}_{2}$ of agent 2 that converges to its nominal position $\overline{\mathbf{x}}_{2}$. Until $t=13 \mathrm{~s}$, the agent 4 behaves the same way. But at this time instant, a sensor fault occurs for this agent and a constant bias $\mathbf{r}_{4}=\left[\begin{array}{ll}-0.2 & 0.2\end{array}\right]^{\top}$ appears on the measurement $\mathbf{y}_{4}$. As the top plots in Fig. 4 and Fig. 3 show, the real position $\mathrm{x}_{4}$ of the agent 4 converges to $\overline{\mathbf{x}}_{4}+\mathbf{r}_{4}$.

The considered measurement bias introduces oscillations on the difference between the estimated and the nominal positions of the agent 4 , i.e. $\widehat{\mathbf{x}}_{4}-\overline{\mathbf{x}}_{4}$. Such a behavior induces oscillations on the input signal of the UAV's rotors and on the agent's movement as illustrated in Fig. 4. However, on a real system, the dynamics of the rotor limits this behavior, slowing down the movement of the agent. For the other agents, the input signal converges to the equilibrium value 

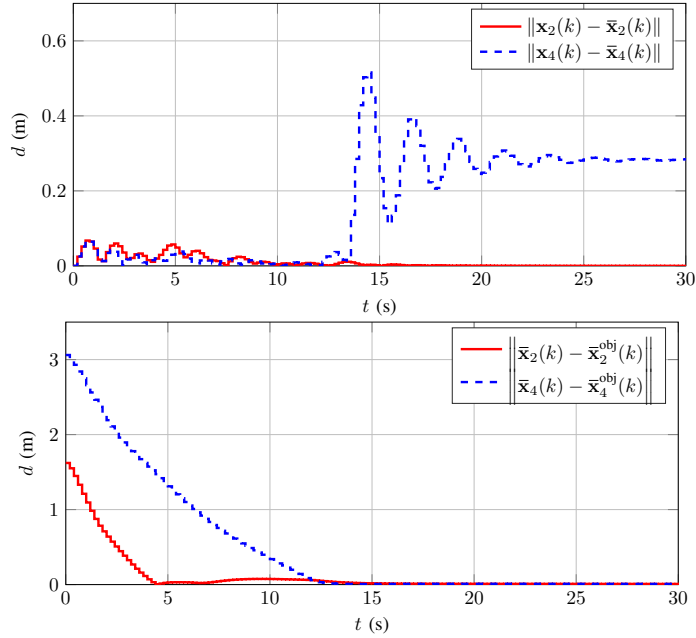

Fig. 4. Distances between the real position of the agents 2 and 4 and their nominal positions (top) and between the nominal positions and the Chebyshev centers (bottom).

$\mathbf{U}_{\alpha}^{\mathrm{eq}}$, with $\alpha \in \overline{1,3}$, with a similar behavior to the agent 2 .

\section{CONCLUSION}

This paper presents a decentralized control algorithm to deploy a multi-agent system in a bounded convex twodimensional area when the position measurement is subject to a bias bounded by a rectangle. The proposed algorithm relies on the guaranteed Voronoi diagram of the multi-agent system and a robust tube-based model predictive control. At each time instant, the guaranteed Voronoi diagram of the rectangles centered on the nominal positions of the agents is computed and the agents track the Chebyshev center of their cells. With the considered decentralized tubebased model predictive control strategy, each agent's position is guaranteed to remain inside its guaranteed Voronoi cell (offering anti collision guarantee) and the multi-agent system converges towards a static Chebyshev configuration, despite the possible uncertain measurements.

Future work will extend this result for bounded perturbations both on the state and on the measurements. Moreover, this strategy will be applied to systems subject to actuator faults. In addition, this control algorithm will be applied to a real fleet of unmanned aerial vehicles.

\section{ACKNOWLEDGMENT}

This work was supported by the LIA of the CNRS on Information, Learning and Control and the Natural Sciences and Engineering Research Council of Canada.

\section{REFERENCES}

[1] H. G. Tanner, A. Jadbabaie, and G. J. Pappas, "Flocking in fixed and switching networks," IEEE Transactions on Automatic Control, vol. 52, no. 5, pp. 863-868, 2007.

[2] R. M. Murray, "Recent research in cooperative control of multivehicle systems," Journal of Dynamic Systems, Measurement, and Control, vol. 129 , no. 5 , pp. 571-583, 2007

[3] F. Bullo, J. Cortés, and S. Martínez, Distributed Control of Robotic Networks, ser. Applied Mathematics Series. Princeton University Press, 2009 .
[4] G. Voronoï, "Nouvelles applications des paramètres continus à la théorie des formes quadratiques," Journal für die reine und angewandte Mathematik, vol. 134, pp. 198-287, 1908.

[5] J. Cortés, S. Martínez, T. Karatas, and F. Bullo, "Coverage control for mobile sensing networks," IEEE Transactions on Robotics and Automation, vol. 20, no. 2, pp. 243-255, 2004.

[6] M. Moarref and L. Rodrigues, "An optimal control approach to decentralized energy-efficient coverage problems," IFAC Proceedings Volumes, vol. 47, no. 3, pp. 6038-6043, 2014.

[7] F. Sharifi, A. Chamseddine, H. Mahboubi, Y. Zhang, and A. G. Aghdam, "A distributed deployment strategy for a network of cooperative autonomous vehicles," IEEE Transactions on Control Systems Technology, vol. 23, no. 2, pp. 737-745, 2015.

[8] M. T. Nguyen, C. S. Maniu, and S. Olaru, "Optimization-based control for multi-agent deployment via dynamic Voronoi partition," IFAC PapersOnLine, vol. 50, no. 1, 2017.

[9] T. Chevet, C. Stoica Maniu, C. Vlad, and Y. Zhang, "Voronoibased UAVs formation deployment and reconfiguration using MPC techniques," in 2018 International Conference on Unmanned Aircraft Systems, 2018, pp. 9-14.

[10] J. Hatleskog, S. Olaru, and M. Hovd, "Voronoi-based deployment of multi-agent system," in 57th Conference on Decision and Control, 2018, pp. 5403-5408.

[11] W. Evans and J. Sember, "Guaranteed Voronoi diagrams of uncertain sites," in 20th Canadian Conference on Computational Geometry, 2008, pp. 207-210

[12] S. Papatheodorou, Y. Stergiopoulos, and A. Tzes, "Distributed area coverage control with imprecise robot localization," in 24th Mediterranean Conference on Control and Automation, 2016, pp. 214-219.

[13] W. Ren and R. W. Beard, Distributed consensus in multi-vehicle cooperative control. Springer, 2008.

[14] M. T. Nguyen and C. S. Maniu, "Voronoi based decentralized coverage problem: From optimal control to model predictive control," in 24th Mediterranean Conference on Control and Automation, 2016, pp 1307-1312.

[15] A. Filotheou, A. Nikou, and D. V. Dimarogonas, "Decentralized control of uncertain multi-agent systems with connectivity maintenance and collision avoidance," in 2018 European Control Conference, 2018 pp. $8-13$

[16] D. Q. Mayne, S. V. Raković, R. Findeisen, and F. Allgöwer, "Robust output feedback model predictive control of constrained linear systems," Automatica, vol. 42, no. 7, pp. 1217-1222, 2006.

[17] D. Limon, I. Alvarado, T. Alamo, and E. F. Camacho, "Robust tubebased MPC for tracking of constrained linear systems with additive disturbances," Journal of Process Control, vol. 20, no. 3, 2010.

[18] F. Blanchini and S. Miani, Set-theoretic Methods in Control. Springer, 2007.

[19] S. V. Raković, E. C. Kerrigan, K. I. Kouramas, and D. Q. Mayne, "Invariant approximations of the minimal robust positively invariant set," IEEE Transactions on Automatic Control, vol. 50, no. 3, pp. 406410, 2005.

[20] I. Prodan, S. Olaru, C. Stoica, and S.-I. Niculescu, "Predictive control for tight group formation of multi-agent systems," IFAC Proceedings Volumes, vol. 44, no. 1, pp. 138-143, 2011.

[21] M. Turanli and H. Temeltas, "Adaptive coverage control with guaranteed power Voronoi diagrams," in 4th International Conference on Systems and Informatics, 2017, pp. 7-13.

[22] H. Eggleston, Convexity. Cambridge University Press, 1958.

[23] Z. Liu, C. Yuan, and Y. Zhang, "Active fault-tolerant control of unmanned quadrotor helicopter using linear parameter varying technique," Journal of Intelligent \& Robotic Systems, vol. 88, no. 2-4, pp 415-436, 2017.

[24] B. Wang, K. A. Ghamry, and Y. Zhang, "Trajectory tracking and attitude control of an unmanned quadrotor helicopter considering actuator dynamics," in 35th Chinese Control Conference, 2016, pp. $10795-10800$.

[25] S. Olaru, J. A. De Doná, M. M. Seron, and F. Stoican, "Positive invariant sets for fault tolerant multisensor control schemes," International Journal of Control, vol. 83, no. 12, pp. 2622-2640, 2010.

[26] J. Mattingley and S. Boyd, "CVXGEN: A code generator for embedded convex optimization," Optimization and Engineering, vol. 13 no. 1, pp. 1-27, 2012.

[27] M. Herceg, M. Kvasnica, C. N. Jones, and M. Morari, "Multiparametric toolbox 3.0," in European Control Conference, 2013, pp. $502-510$. 